\title{
How Multiphoton and Harmonic Generation Microscopy Methods are Useful in Understanding Lung Structure and Related Diseases
}

\author{
Thomas Abraham,* Jeremy A. Hirota, Mark Elliot, Darryl Knight, James Hogg \\ The James Hogg Research Centre, Heart and Lung Institute at St. Paul's Hospital-University of \\ British Columbia, 1081 Burrard Street, Vancouver, Canada V6Z 1Y6. \\ thomas.abraham@hli.ubc.ca
}

A minimally invasive imaging method which can provide both cellular and extracellular structural details with sufficient specificity, sensitivity and spatial resolution is particularly useful for understanding the lung structure and related diseases. Multiphoton microscopy, which uses ultrashort IR laser pulses as the excitation source, is efficient in producing multiphoton excitation fluorescence (MPEF) signals from endogenously fluorescent macromolecular systems (e.g. elastin) and in the induction of highly specific second harmonic generation (SHG) signals from noncentrosymmetric macromolecules such as fibrillar collagens from lung tissues [1-3]. Since the SHG emission wavelength is relatively shorter than that of MPEF, both these signals can be captured simultaneously to provide spatially resolved 3D structural organization of extracellular matrix as well as the cellular morphologies with spectral specificity in their native environments. These imaging modalities are minimally invasive since the structures deep within tissues $(\sim 0.5$ millimeter $)$ can be visualized without the need for fixation, sectioning or staining. This presentation focuses on specific applications of these high resolution microscopy methods in lung structural imaging, particularly morphological features of alveolar and airway structures, visualizing and quantifying extracellular matrix remodeling accompanying emphysematous destructions in human lungs, as well as small animal models of asthma. Flash frozen cryo-matrix embedded lung samples were first immobilized on the flat bottom of a small dish, immersed in phosphate buffed saline (PBS) solution and sub-sequential imaging operations were performed directly on these unfixed, unstained tissue sections using high resolution water immersion objectives. In reflection geometry, non-de-scanned detectors and spectral scanning mode were used for generating the 3D images and for the spectra, respectively. An infrared femto-second laser pulses tuned to 880-nm was used for generating SHG and MPEF images. From the simultaneously captured spectrally distinguishable SHG and MPEF signals generated from the human lung tissues, we have shown that structural dissociation of relatively extendable elastic fibers and relatively stiffer fibrillar collagens in the alveolar walls occurs in the early lesions of emphysematous diseases (Fig.1). Comparison of house dust mite exposed mouse lung tissue (which is a small animal model of asthma) with control saline exposure showed high degree of structural remodeling of the airway with obvious changes in the spatial arrangements of fibrillar collagen and other ECM component structures (Fig.1). In addition, these MPEF airway images showed considerable accumulation of endogenously fluorescent round shaped objects around the airways, which may be inflammatory cells consistent with airway inflammation. The 3D mapping of lung ECM components, and the specificity of collagen (SHG) also allowed computation of relative changes of fibrillar collagen and other endogenously fluorescent components associated with lung tissues undergoing structural remodeling. Our research efforts in this area conclude that the specificity, sensitivity, spatial resolution, and minimally invasive nature of probing extracellular matrix structures in lung tissues in their native states make these multiphoton based microscopy methods superior to any other currently available conventional microscopy methods. 


\section{Reference:}

[1] Abraham, T., Hogg, J. Journal of Structural Biology 171 (2010) 189-196.

[2] Abraham, T., Hogg, J. Proceedings of SPIE, 7555 (2010) 75550X1-75550X9.

[3] Abraham, T., Wadsworth, S., Carthy, J., Petchkovski, D., McManus, B. Respirology 16 (2011) $22-33$.
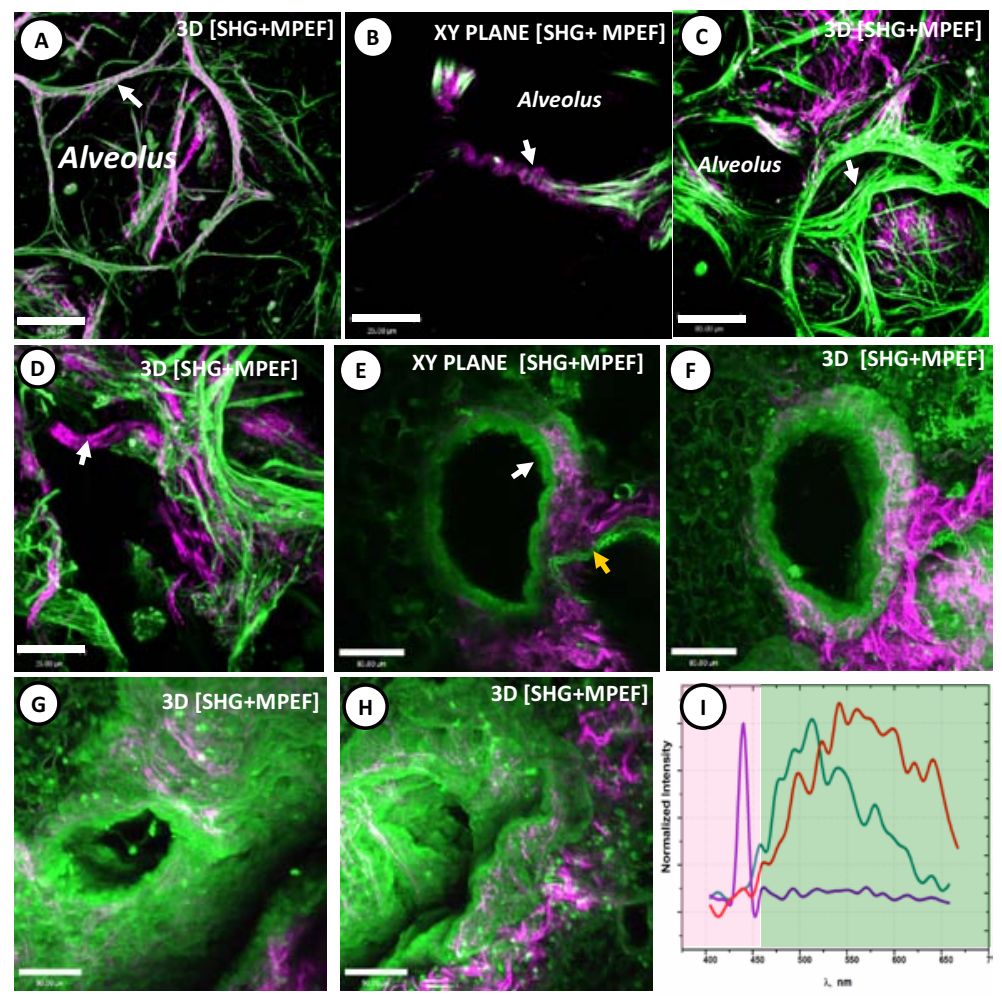

Figure 1: Representative 3D SHG image (violet color) originating from the fibrillar collagen overlaid with the MPEF image (green color) originating from the endogenously fluorescent ECM components of healthy alveolar tissues in their native states is shown in A [Scale bar: $80 \mathrm{~mm}$ ]. The collagen appeared to be in the form of fused band-like structures (violet color) which is condensed around the alveolar walls. The lung elastin (green color) consisted of fine fibres of varying thickness and also appeared to be fused to form band-like structure. These structural features, plus the spirally wound form of collagen band around the elastic fibres can be clearly seen in B [Scale bar: $25 \mathrm{~mm}$ ]. The similar images (C and D) obtained from the emphysematous lung shows a highly

disorganized alveolar wall structure. Both collagen and elastin exhibited a grossly altered configuration in these emphysematous lung samples and the size of the ECM structures, particularly collagen, showed considerable variations as evident from highly optically magnified image (see white arrow in D). Similar small airway images generated from control mouse lung tissue is shown in E and F. As shown, the major ECM component fibrillar collagens (violet color) lie beneath the endogenously fluorescent epithelium (green color, see white arrow in E) and other endogenously fluorescent ECM components. A blood vessel, which is characterized by an inner lining of wavelike elastic fibers (green color) surrounded by fibrillar collagen is also seen (see yellow arrow in E). Similar images of lung airway tissues obtained from a mouse exposed to house dust mite (small animal asthma model) are shown in $\mathrm{G}$ and $\mathrm{H}$. The airway walls of these lung tissues appeared to be much thicker compared to the normal ones. These MPEF images also show considerable accumulation of endogenously fluorescent round shaped objects (green color) around the airways. A spectral scan data of alveolar wall is shown in I. The measured SHG signal (violet line) is manifested by a narrow Gaussian peak and is spectrally clean, arising only at the half of the excitation wavelength $880 \mathrm{~nm}$. The measured broadband emission spectra of elastin (green line) and the round shaped objects in the alveolar area (red line) are also shown. 